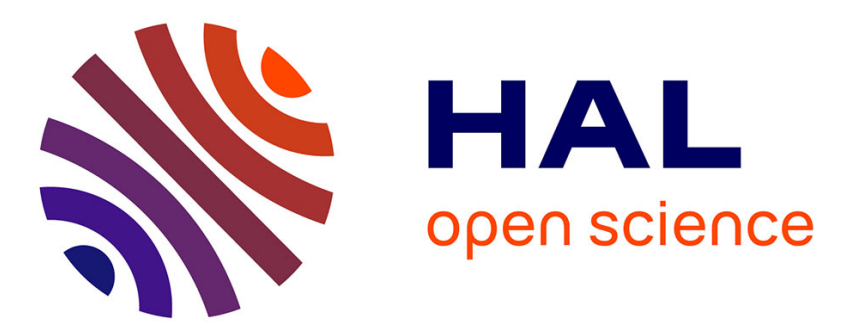

\title{
Algebraic parameter estimation of a biased sinusoidal waveform signal from noisy data
}

Rosane Ushirobira, Wilfrid Perruquetti, Mamadou Mboup, Michel Fliess

\section{To cite this version:}

Rosane Ushirobira, Wilfrid Perruquetti, Mamadou Mboup, Michel Fliess. Algebraic parameter estimation of a biased sinusoidal waveform signal from noisy data. Sysid 2012, 16th IFAC Symposium on System Identification, Jul 2012, Brussels, Belgium. hal-00685067

\section{HAL Id: hal-00685067 https://hal.inria.fr/hal-00685067}

Submitted on 3 Apr 2012

HAL is a multi-disciplinary open access archive for the deposit and dissemination of scientific research documents, whether they are published or not. The documents may come from teaching and research institutions in France or abroad, or from public or private research centers.
L'archive ouverte pluridisciplinaire HAL, est destinée au dépôt et à la diffusion de documents scientifiques de niveau recherche, publiés ou non, émanant des établissements d'enseignement et de recherche français ou étrangers, des laboratoires publics ou privés. 


\title{
Algebraic parameter estimation of a biased sinusoidal waveform signal from noisy data
}

\author{
Rosane Ushirobira* Wilfrid Perruquetti ${ }^{* *}$ \\ Mamadou Mboup ${ }^{* * *}$ Michel Fliess ${ }^{* * * *}$ \\ * Institut de Mathématiques de Bourgogne (CNRS), Université de \\ Bourgogne \&3 Non-A Inria Lille - Nord Europe, France (e-mail: \\ Rosane.Ushirobira@inria.fr) \\ ** Université Lille Nord de France $\& 3$ École Centrale de Lille $\& 3$ Non-A \\ Inria Lille - Nord Europe 83 LAGIS (CNRS), France (e-mail: \\ Wilfrid.Perruquetti@inria.fr) \\ *** CReSTIC, Université de Reims Champagne Ardenne, France 8 \\ Non-A Inria Lille - Nord Europe (e-mail: \\ Mamadou.Mboup@univ-reims.fr) \\ **** LIX (CNRS), École polytechnique, 91128 Palaiseau (e-mail: \\ Michel.Fliessopolytechnique.edu) \\ \& AL.I.E.N., 24-30 rue Lionnois, BP 60120, 54003 Nancy, France, \\ WWW.alien-sas.com
}

\begin{abstract}
The amplitude, frequency and phase of a biased and noisy sum of two complex exponential sinusoidal signals are estimated via new algebraic techniques providing a robust estimation within a fraction of the signal period. The methods that are popular today do not seem able to achieve such performances. The efficiency of our approach is illustrated by several computer simulations.
\end{abstract}

Keywords: Parameter identification, differential algebra, sinusoidal waveform.

\section{INTRODUCTION}

The parameter estimation of a biased sinusoidal signal in a noisy environment is an important issue occurring in many practical engineering problems, e.g. the signal demodulation in communications, the regulation of electronic converters power, the circadian rhythm of biological cells and the modal identification for flexible structures (see Trapero et al. [2007b]).

Many different resolution methods have been developed, such as linear or nonlinear regression, subspace methods (Haykin [1991], Roy et al. [1989], Kahn et al. [1992]), the extended Kalman filter (Bittanti et al. [2000]), the notches filter (Regalia et al. [1995]), or alternatively, the use of techniques borrowed from adaptive nonlinear control (Hsu et al. [1999], Mojiri et al. [2004]). However, the robust parameter estimation in a fraction of the time signal, in the presence of noise and of an unknown constant bias, is not yet fully solved. This paper draws its inspiration from the algebraic analysis of Fliess et al. [2003, 2010, 2008], Fliess [2008], Mboup [2009]. In addition to numerical simulations found in these papers, we refer to Neves et al. [2006], Trapero et al. [2007a,b, 2008b] for more very encouraging results in concrete examples.

In his 1795 seminal paper, Riche de Prony studies the parameter estimation of a finite sum of sinusoidal functions (see Riche de Prony [1795], Kahn et al. [1992], Osborne et al. [1995]). In this paper, we are interested in Prony's problem for a two-terms sum of complex sinusoidal func- tions, meaning that our aim is to estimate the parameters of the signal (see also Neves et al. [2007] for a quite related study)

$$
x(t)=\alpha_{1} \exp i\left(\omega_{1} t+\phi_{1}\right)+\alpha_{2} \exp i\left(\omega_{2} t+\phi_{2}\right)
$$

from the biased and noisy output measure

$$
y(t)=x(t)+\beta+\varpi
$$

where $\beta$ is an unknown constant bias and $\varpi$ is a noise ${ }^{1}$.

A linear parametric estimation problem may often be formalized as finding a good approximation of some vector $\Theta$ on the basis of an observed signal that is a linear functional of the true signal depending on a set of parameters and a noise corrupting the observation. Here the signal $z(t)=x(t)+\beta$ and $\Theta$ are linearly differentially algebraic. Indeed, we have the following differential equation:

$$
\ddot{z}(t)-i\left(\omega_{1}+\omega_{2}\right) \dot{z}(t)-\omega_{1} \omega_{2}(z(t)-\beta)=0 .
$$

Notice that the signal frequencies appear as roots of the characteristic equation of the above relation which is also behind Prony's method and other techniques such as adaptive notch filters: take this equation as the numerator of some transfer function, then the filter zeros align with the signal frequencies. The further applied algebraic operator will take advantage of this remark.

In the operational domain, we obtain

\footnotetext{
1 We use here the framework developed in Fliess [2006, 2008]. It is independent of any probabilistic modeling. In this point of view, the noise should be viewed as fast oscillations (see Fliess [2006]).
} 


$$
\begin{aligned}
& s\left(s-i \omega_{1}\right)\left(s-i \omega_{2}\right) Z(s)= \\
& \quad s\left(s-i\left(\omega_{1}+\omega_{2}\right)\right) z(0)+s \dot{z}(0)-\beta \omega_{1} \omega_{2} .
\end{aligned}
$$

Among the unknown parameters, we wish to estimate $\Theta_{\text {est }}:=\left\{\theta_{1}, \theta_{2}, \theta_{3}, \theta_{4}\right\}$, but not the bias $\Theta_{\overline{\mathrm{est}}}=\left\{\theta_{5}\right\}$ :

$$
\begin{aligned}
& \theta_{1}=-\omega_{1} \omega_{2}, \quad \theta_{2}=-i\left(\omega_{1}+\omega_{2}\right), \\
& \theta_{3}=-x(0)=\beta-z(0), \quad \theta_{4}=-\dot{z}(0)=-\dot{x}(0), \\
& \theta_{5}=-\beta
\end{aligned}
$$

Notice that the parameters $\alpha_{1}, \omega_{1}, \phi_{1}, \alpha_{2}, \omega_{2}, \phi_{2}$ can be easily deduced from $\Theta_{\text {est }}$, see Lemma 15 in the Appendix.

Using the notation (3)-(5), the equation (2) reads as

$$
\begin{aligned}
\left(s^{3}+\theta_{2} s^{2}+\theta_{1} s\right) Z(s)+\left(s^{2}+\theta_{2} s\right) \theta_{3}+\theta_{4} s+ \\
\left(s^{2}+\theta_{2} s+\theta_{1}\right) \theta_{5}=0 .(6)
\end{aligned}
$$

From (6) we would like to obtain a system of equations on $\Theta_{\text {est }}$ and independent of $\Theta_{\overline{\text { est }}}$. We consider the algebraic extensions $\mathbb{C}_{\Theta}:=\mathbb{C}(\Theta), \mathbb{C}_{\Theta_{\text {est }}}:=\mathbb{C}\left(\Theta_{\text {est }}\right)$ and $\mathbb{C}_{\Theta \overline{\text { est }}}:=\mathbb{C}\left(\Theta_{\overline{\text { est }}}\right)$. Moreover, $\mathbb{C}_{\Theta}[s]$ (respectively $\mathbb{C}_{\Theta_{\text {est }}}[s]$, $\left.\mathbb{C}_{\Theta \overline{e s t}}[s]\right)$ denotes the polynomial ring in the variable $s$ with coefficients in $\mathbb{C}_{\Theta_{\text {est }}}$ (respectively in $\mathbb{C}_{\Theta_{\overline{\text { est }}}}$ ). We obtain the relation:

$$
\begin{gathered}
\mathcal{R}\left(s, Z(s), \Theta_{\text {est }}, \Theta_{\overline{\text { est }}}\right):=P(s) Z(s)+Q(s)+\bar{Q}(s)=0 \\
\text { with } P(s)=s T(s) \text { and } \\
\qquad(s)=s^{2} \theta_{3}+s\left(\theta_{4}+\theta_{2} \theta_{3}\right) \in \mathbb{C}_{\Theta_{\text {est }}}[s] \\
\text { and } \bar{Q}(s)=T(s) \theta_{5} \in \mathbb{C}_{\Theta \overline{\text { est }}}[s],
\end{gathered}
$$

where we set $T(s)=s^{2}+\theta_{2} s+\theta_{1} \in \mathbb{C}_{\Theta_{\text {est }}}[s]$.

Now, we proceed in three steps:

(1) Algebraic elimination of all terms in $\Theta_{\overline{\mathrm{est}}}$ : all differential operators that annihilate $\bar{Q}$ can be generated by a single operator in $\mathbb{C}_{\Theta_{\text {est }}}(s)\left[\frac{d}{d s}\right]^{2}$, called a $\bar{Q}$-minimal annihilator. These annihilators will be rewritten in a canonical form.

(2) Obtaining a system of equations on $\Theta_{\text {est }}$ : we apply on $\mathcal{R}$ several differential operators annihilating $\bar{Q}$. Using their canonical forms, choices will be made to obtain a system of equations with good numerical properties (once back in the time domain).

(3) Resolution of the obtained system: we use the inverse Laplace transform

$$
\mathcal{L}^{-1}\left(\frac{1}{s^{m}} \frac{d^{p} Z(s)}{d s^{p}}\right)=\frac{(-1)^{p} t^{m+p}}{(m-1) !} \int_{0}^{1} w^{m-1, p}(\tau) z(t \tau) d \tau
$$

with $w^{m, p}(t)=(1-t)^{m} t^{p}, \forall p, m \in \mathbb{N}, m \geq 1$ to bring the equations back in the time domain. The integers $m, p$ will be chosen as small as possible so that the resulting estimation is as least as possible sensitive to the noise.

The first point emphasizes the central role played by $\bar{Q}$ minimal annihilators. They are defined in Section 2 where we describe the algebraic structure behind them and the algebraic elimination technique to eliminate $\Theta_{\overline{\text { est }}}$ (subsection 2.1). Minimal annihilators can be rewritten in a

2 The polynomial ring in $\frac{d}{d s}$ with coefficients in $\mathbb{C}_{\Theta_{\text {est }}}[s]$ canonical form, detailed in subsection 2.1. The parameter estimation is provided in section 3. Section 4 contains convincing numerical experiments that illustrate our techniques and are easily implementable. These experiments are compared to the well-known modified Prony's method.

\section{AN ALGEBRAIC FRAMEWORK FOR OBTAINING ANNIHILATORS}

The algebraic framework is borrowed from Fliess et al. [2010, 2003, 2008], Fliess [2008], Mboup [2009] ${ }^{3}$. More details about the algebraic notions can be found in Dixmier et al. [1974] and McConnell et al. [2000].

Recall that we wish to annihilate

$$
\bar{Q}=\left(s^{2}+\theta_{2} s+\theta_{1}\right) \theta_{5} \in \mathbb{C}_{\Theta \overline{\text { est }}}[s] \text {. }
$$

Since we have a polynomial in $s$, the natural idea is to look for operator in $\frac{d}{d s}$. It is clear that operators of order 4 greater than 3 will annihilate $\bar{Q}$, e.g. $\Pi_{1}=\left(s \frac{d}{d s}-2\right) \circ\left(s \frac{d}{d s}-\right.$ $1) \circ\left(s \frac{d}{d s}\right)$ and $\Pi_{2}=\frac{d^{3}}{d s^{3}}$. One question would be whether these annihilators are the same. Another question would be whether there exists a lower order annihilator. Answers are provided by the algebraic structure of the Weyl algebra $\mathbb{C}_{\Theta}(s)\left[\frac{d}{d s}\right]$.

\subsection{Weyl algebra}

We consider $\mathbb{K}$ a field of characteristic zero (here $\mathbb{K}=\mathbb{C}$ ). Definition 1. Let $k \in \mathbb{N} \backslash\{0\}$. The Weyl algebra $\mathrm{A}_{k}(\mathbb{K})$ is the $\mathbb{K}$-algebra generated by $p_{1}, q_{1}, \ldots, p_{k}, q_{k}$ satisfying the relations

$$
\left[p_{i}, q_{j}\right]=\delta_{i j},\left[p_{i}, p_{j}\right]=\left[q_{i}, q_{j}\right]=0, \forall 1 \leq i, j \leq k
$$

where $[\cdot, \cdot]$ is the commutator defined by $[u, v]:=u v-v u$, $\forall u, v \in \mathrm{A}_{k}(\mathbb{K})$. We will simply write $\mathrm{A}_{k}$ instead of $\mathrm{A}_{k}(\mathbb{K})$ when we do not need to make explicit the base field.

A well-known fact is that $A_{k}$ can be realized as the algebra of polynomial differential operators on $\mathbb{K}\left[s_{1}, \ldots, s_{k}\right]$ with

$$
p_{i}=\frac{\partial}{\partial s_{i}} \text { and } q_{i}=s_{i} \times, \forall 1 \leq i \leq k .
$$

We can also write

$$
\mathrm{A}_{k}=\mathbb{K}\left[q_{1}, \ldots, q_{k}\right]\left[p_{1}, \ldots, p_{k}\right]=\mathbb{K}\left[s_{1}, \ldots, s_{k}\right]\left[\frac{\partial}{\partial s_{1}}, \ldots, \frac{\partial}{\partial s_{k}}\right]
$$

(notice that we use the same notation for the variable $s_{i}$ and for the operator multiplication by $s_{i}$ here).

There is a closely related algebra to $A_{k}$ : it is defined as the set of differential operators on $\mathbb{K}\left[s_{1}, \ldots, s_{k}\right]$ with coefficients in the rational functions field $\mathbb{K}\left(s_{1}, \ldots, s_{k}\right)$. We denote it by $\mathrm{B}_{k}(\mathbb{K})$ and write

$$
\mathrm{B}_{k}(\mathbb{K}):=\mathbb{K}\left(q_{1}, \ldots, q_{k}\right)\left[p_{1}, \ldots, p_{k}\right]=\mathbb{K}\left(s_{1}, \ldots, s_{k}\right)\left[\frac{\partial}{\partial s_{1}}, \ldots, \frac{\partial}{\partial s_{k}}\right]
$$

Sometimes we will simply write $\mathrm{B}_{k}$ instead of $\mathrm{B}_{k}(\mathbb{K})$. In the case $k=1$ for instance, we have

$$
\mathrm{A}_{1}=\langle p, q \mid p q-q p=1\rangle=\mathbb{K}[s]\left[\frac{d}{d s}\right] \text { and } \mathrm{B}_{1}=\mathbb{K}(s)\left[\frac{d}{d s}\right]
$$

3 Similar tools were also used for numerical differentiation of noisy signal (see Mboup et al. [2009], Liu et al. [2011]) and change-point detection (see Fliess et al. [2010]).

4 The order of an operator $\Pi \in \mathbb{C}_{\Theta}(s)\left[\frac{d}{d s}\right]$ is its degree as a polynomial in the variable $\frac{d}{d s}$. 
Proposition 2. A basis for $\mathrm{A}_{k}$ is given by $\left\{q^{I} p^{J} \mid I, J \in \mathbb{N}^{k}\right\}$ where $q^{I}:=q_{1}^{i_{1}} \ldots q_{k}^{i_{k}}$ and $p^{J}:=p_{1}^{j_{1}} \ldots p_{k}^{j_{k}}$.

An element $F \in \mathrm{A}_{k}$ can be written in its canonical form,

$$
F=\sum_{I, J} \lambda_{I J} q^{I} p^{J} \text { with } \lambda_{I J} \in \mathbb{K} .
$$

Example 3. In this paper the following identities are useful:

$$
\begin{aligned}
& q^{m} p^{n}=p^{n} q^{m}+\sum_{k=1}^{m}\left(\begin{array}{c}
m \\
k
\end{array}\right)\left(\begin{array}{l}
n \\
k
\end{array}\right) k !(-1)^{k} p^{n-k} q^{m-k} \\
& p^{n} q^{m}=q^{m} p^{n}+\sum_{k=1}^{n}\left(\begin{array}{c}
n \\
i
\end{array}\right)\left(\begin{array}{c}
m \\
i
\end{array}\right) i ! q^{m-i} p^{n-i}
\end{aligned}
$$

Similarly, an element $F \in \mathrm{B}_{k}$ can be written as

$$
F=\sum_{I} g_{I}(s) p^{I} \text { with } g_{I}(s) \in \mathbb{K}\left(s_{1}, \ldots, s_{k}\right) .
$$

The order of an element $F \in \mathrm{B}_{k}, F=\sum_{I} g_{I}(s) p^{I}$ is defined as

$$
\operatorname{ord}(F):=\max \left\{\left[I|| g_{I}(s) \neq 0\right\} .\right.
$$

Notice that the same definition is valid for the Weyl algebra $A_{k}$ since $A_{k} \subset B_{k}$.

Proposition 4. $\mathrm{A}_{k}$ is a domain. Moreover, $\mathrm{A}_{k}$ is simple and Noetherian.

However, $A_{k}$ is neither a principal right domain, nor a principal left domain.

Proposition 5. For the algebra $\mathrm{B}_{k}$, one has:

(1) $\mathrm{B}_{k}$ is a domain. Moreover, $\mathrm{B}_{k}$ is simple and Noetherian.

(2) $\mathrm{B}_{1}$ admits a left division algorithm, that is, if $F$, $G \in \mathrm{B}_{1}$, then there exists $q, r \in \mathrm{B}_{1}$ such that $F=q G+r$ and $\operatorname{ord}(r)<\operatorname{ord}(G)$. As a consequence, $\mathrm{B}_{1}$ is a principal left domain.

Since $\frac{d}{d s}$ is a derivation operator we have:

Proposition 6. (Derivation). For $F \in \mathbb{C}[s]$ and $X(s)$ the Laplace transform of a signal $x(t)$, we have (Leibniz rule):

$$
\frac{d^{n}}{d s^{n}}(F \quad X)=\sum_{k=0}^{n}\left(\begin{array}{l}
n \\
k
\end{array}\right) \frac{d^{k} F}{d s^{k}} \frac{d^{n-k} X}{d s^{n-k}}
$$

\subsection{Annihilator}

In the sequel, to distinguish whether an annihilator depends on $\Theta_{\text {est }}$ or not, we work with $\mathrm{B}:=\mathrm{B}_{1}=\mathbb{C}(s)\left[\frac{d}{d s}\right]$ and $\mathrm{B}_{\Theta_{\text {est }}}:=\mathbb{C}_{\Theta_{\text {est }}}(s)\left[\frac{d}{d s}\right]$.

Definition 7. Let $R \in \mathbb{C}_{\Theta}[s]$. Consider the left ideal:

$$
\operatorname{Ann}_{\mathrm{B}}(R)=\{F \in \mathrm{B} \mid F(R)=0\} .
$$

A $R$-annihilator w.r.t. $\mathrm{B}$ is an element $\Pi \in \operatorname{Ann}_{\mathrm{B}}(R)$.

Remark 8. Let us note that $\operatorname{Ann}_{\mathrm{B}}(R)$ is a left principal ideal. Thus it is generated by a single generator $\Pi_{\min } \in \mathrm{B}$ called a minimal $R$-annihilator w.r.t. B. So

$$
\operatorname{Ann}_{\mathrm{B}}(R)=\mathrm{B} \Pi_{\text {min }} \text {. }
$$

Remark that $\operatorname{Ann}_{\mathrm{B}}(R)$ contains annihilators in finite integral form, i.e. operators with coefficients in $\mathbb{C}\left[\frac{1}{s}\right]$.
Remark 9. Remark 8 still holds if we replace $\mathrm{B}$ by $\mathrm{B}_{\Theta_{\text {est }}}$ in Definition 7. That means that $\operatorname{Ann}_{\mathrm{B}_{\Theta}}(R)$ is generated by a unique generator $\Pi_{\text {min }} \in B_{\Theta_{\text {est }}}$ (up to multiplication by a polynomial in $\mathbb{C}_{\Theta_{\text {est }}}(s)$ ) called a minimal $R$-annihilator w.r.t. $\mathrm{B}_{\Theta_{e s t}}$.

We have the following lemmas:

Lemma 10. Let $Q_{n}=s^{n}, n \in \mathbb{N}$. A minimal $Q_{n^{-}}$ annihilator is

$$
\Pi_{n}=s \frac{d}{d s}-n
$$

(unique up to a multiplication by a polynomial in $\mathbb{C}(s)$ ).

Let us note that for $m, n \in \mathbb{N}$, the operators $\Pi_{m}$ and $\Pi_{n}$ commute. Thus one can take advantage of the following lemma

Lemma 11. Let $P_{1}, P_{2} \in \mathbb{C}_{\Theta \overline{\text { est }}}[s]$. Let $\Pi_{i}$ be a $P_{i^{-}}$ annihilator $(i=1,2)$ such that $\Pi_{1} \Pi_{2}=\Pi_{2} \Pi_{1}$. Then $\Pi_{1} \Pi_{2}$ is a $\left(\mu P_{1}+\eta P_{2}\right)$-annihilator for all $\mu, \eta \in \mathbb{C}_{\Theta \overline{\text { est }}}$.

Using this Lemma and considering $\bar{Q}$ in (7), we obtain a minimal $\bar{Q}$-annihilator w.r.t. $\mathrm{B}$ where $\bar{Q}=$ $\left(s^{2}+\theta_{2} s+\theta_{1}\right) \theta_{5} \in \mathbb{C}_{\Theta \overline{\text { est }}}[s]:$

$$
\Pi_{\min }=\left(s \frac{d}{d s}-2\right) \circ\left(s \frac{d}{d s}-1\right) \circ\left(s \frac{d}{d s}\right) .
$$

The identities in Example 3 give:

$$
\Pi_{\min }=s^{3} \frac{d^{3}}{d s^{3}} .
$$

Lemma 12. Let $R \in \mathbb{C}_{\Theta_{\text {est }}}[s]$. Then a minimal $R$ annihilator w.r.t $\mathrm{B}_{\Theta_{\text {est }}}$ is

$$
\Pi_{\min }=R \frac{d}{d s}-\frac{d R}{d s} .
$$

In our example, by Lemma 12 we obtain that the $\bar{Q}$ annihilator w.r.t. $\mathrm{B}_{\Theta_{\mathrm{est}}}$ is:

$$
\Pi_{\mathrm{min}}^{\Theta_{\mathrm{est}}}=\left(s^{2}+\theta_{2} s+\theta_{1}\right) \frac{d}{d s}-\left(2 s+\theta_{2}\right) .
$$

\section{PARAMETER ESTIMATION}

Our estimation problem is equivalent to find a family of annihilators $\left(\Pi_{i}\right)_{i=1}^{r}$ in $\mathbb{C}(s)\left[\frac{d}{d s}\right]$ that applied on $(7)$ provides a set of equations enabling the computation of $\Theta_{\text {est }}$ in the time domain. This family will be generated by a minimal $\bar{Q}$-annihilator.

Formula (8) justifies the use of finite-integral form annihilators. Moreover, these operators should be of minimal degree in $\frac{d}{d s}$ to minimize noise sensitivity. Lastly, the obtained system of equations should be well-balanced to provide good numerical estimation.

Recall that the minimal $\bar{Q}$-annihilator $\Pi_{\min }$ is a generator of the ideal $\operatorname{Ann}_{\mathrm{B}}(\bar{Q})$, so a general $\bar{Q}$-annihilator will be of the form:

$$
\Pi=\left(\sum_{i=0}^{\ell} g_{i}(s) \frac{d^{i}}{d s^{i}}\right) \circ \Pi_{\min }
$$

where $g_{i}(s) \in \mathbb{C}(s), \forall i=0, \ldots, \ell$. 


\subsection{Estimation of $\theta_{1}$ and $\theta_{2}$}

Applying $\Pi_{\min }=s^{3} \frac{d^{3}}{d s^{3}}$, the minimal $\bar{Q}$-annihilator w.r.t. $\mathrm{B}$, on relation (7) gives

$$
\begin{aligned}
\Pi_{\min }(P(s) Z(s))=P_{3}(s) & \frac{d^{3} Z(s)}{d s^{3}}+P_{2}(s) \frac{d^{2} Z(s)}{d s^{2}} \\
& +P_{1}(s) \frac{d Z(s)}{d s}+P_{0}(s) Z(s)
\end{aligned}
$$

where $P_{0}(s)=6 s^{3}, \quad P_{2}(s)=9 s^{5}+6 s^{4} \theta_{2}+3 s^{3} \theta_{1}$, $P_{1}(s)=18 s^{4}+6 s^{3} \theta_{2}, \quad P_{3}(s)=s^{6}+s^{5} \theta_{2}+s^{4} \theta_{1}$, and $\Pi_{\min }(Q(s))=\Pi_{\min }(\bar{Q}(s))=0$.

That provides the following algebraic relation

$$
P_{3}(s) \frac{d^{3} Z(s)}{d s^{3}}+P_{2}(s) \frac{d^{2} Z(s)}{d s^{2}}+P_{1}(s) \frac{d Z(s)}{d s}+P_{0}(s) Z(s)=0 .
$$

We obtain a single equation in $\theta_{1}$ and $\theta_{2}$. To linearly identify these two parameters, we need two independent equations. However, the following result show that this is not possible in the operational domain (see the appendix for a proof):

Theorem 13. There do not exist two $\bar{Q}$-annihilators w.r.t B leading to two independent equations in $\theta_{1}$ and $\theta_{2}$.

Remark 14. Let us note that for a similar parameter identification problem of a single sinusoid, it is indeed possible to find two independent equations in the operational domain (see Ushirobira et al. [2011]).

Therefore we will use such a construction in the time domain. For this, since $\bar{Q}$-annihilators are of the form (11), to get two equations we select $\ell=1$. That leads to the following $4^{t h}$-order annihilator written in the canonical form:

$$
\Pi=g_{0}(s) \frac{d^{3}}{d s^{3}}+g_{1}(s) \frac{d^{4}}{d s^{4}},
$$

where $g_{0}(s), g_{1}(s) \in \mathbb{C}(s)$. The choices of $g_{0}(s)=1$, $g_{1}(s)=0$ and then $g_{0}(s)=0, g_{1}(s)=1$, give two equations in the operational domain leading to the following system in the time domain:

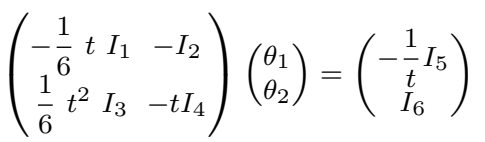

where

$$
\begin{aligned}
& I_{1}=\int_{0}^{1}\left(6 w^{2,3}(\tau)-3 w^{2,2}(\tau)\right) z(t \tau) d \tau \\
& I_{2}=\int_{0}^{1}\left(5 w^{1,3}(\tau)-5 w^{1,2}(\tau)+w^{1,1}(\tau)\right) z(t \tau) d \tau \\
& I_{3}=\int_{0}^{1}\left(7 w^{2,4}(\tau)-4 w^{2,3}(\tau)\right) z(t \tau) d \tau \\
& I_{4}=\int_{0}^{1}\left(-7 w^{1,4}(\tau)+8 w^{1,3}(\tau)-2 w^{1,2}(\tau)\right) z(t \tau) d \tau \\
& I_{5}=\int_{0}^{1}\left(w^{3,0}(\tau)-9 w^{2,1}(\tau)+9 w^{1,2}(\tau)-w^{0,3}(\tau)\right) z(t \tau) d \tau \\
& I_{6}=\int_{0}^{1}\left(-4 w^{3,1}(\tau)+18 w^{2,2}(\tau)-12 w^{1,3}(\tau)+w^{0,4}(\tau)\right) z(t \tau) d \tau
\end{aligned}
$$

The expressions for $\theta_{1}$ and $\theta_{2}$ are thus obtained:

$$
\begin{gathered}
\theta_{1}=-\frac{6}{t^{2}} \frac{\left(-I_{4} I_{5}+I_{6} I_{2}\right)}{I_{4} I_{1}+I_{3} I_{2}} \\
\theta_{2}=\frac{1}{t} \frac{I_{1} I_{6}-I_{3} I_{5}}{I_{4} I_{1}+I_{2} I_{3}}
\end{gathered}
$$

\subsection{Estimation of $\theta_{3}$ and $\theta_{4}$}

Using annihilators generated by the minimal $\bar{Q}$-annihilator w.r.t. B $:=\mathbb{C}(s)\left[\frac{d}{d s}\right]$ given by (10), we could linearly identify the parameters $\theta_{1}$ and $\theta_{2}$. These annihilators do not depend on the parameters to be found. Now, one can show that it is not possible to identify linearly the remaining parameters $\theta_{3}$ and $\theta_{4}$, so we will use nonlinear equations in $\theta_{1}$ and $\theta_{2}$. So, let us consider the $\bar{Q}$-annihilator w.r.t. $\mathrm{B}_{\Theta_{\mathrm{est}}}$ :

$$
\Pi_{\min }^{\Theta_{\text {est }}}=\left(s^{2}+\theta_{2} s+\theta_{1}\right) \frac{d}{d s}-\left(2 s+\theta_{2}\right) .
$$

Applying it on (7) gives:

$$
\begin{aligned}
\Pi_{\min }^{\Theta_{\text {est }}}(P(s) Z(s)) & =T(s)^{2}\left(s \frac{d Z(s)}{d s}+Z(s)\right) \\
\Pi_{\min }^{\Theta_{\text {est }}}(Q(s)) & =-\theta_{4} s^{2}+2 \theta_{1} \theta_{3} s+\theta_{1}\left(\theta_{4}+\theta_{2} \theta_{3}\right) \\
\Pi_{\min }^{\Theta_{\text {est }}}(\bar{Q}(s)) & =0
\end{aligned}
$$

where $T(s)=s^{2}+\theta_{2} s+\theta_{1} \in \mathbb{C}[s]$. That gives the following algebraic relation:

$T(s)^{2}\left(Z(s)+s \frac{d Z(s)}{d s}\right)+\left(\theta_{1}-s^{2}\right) \theta_{4}+\theta_{1}\left(2 s+\theta_{2}\right) \theta_{3}=0$.

We obtain a single equation in $\theta_{3}$ and $\theta_{4}$. As in the previous subsection, we use (11) with $\ell=1$, where $\Pi_{\min }$ is replaced by $\Pi_{\text {min }}^{\Theta_{\text {est }}}$. That leads to the following $4^{\text {th }}$-order annihilator written in the canonical form:

$$
\Pi=g_{0}(s) \Pi_{\mathrm{min}}^{\Theta_{\mathrm{est}}}+g_{1}(s)\left(T(s) \frac{d^{2}}{d s^{2}}-2\right),
$$

where $g_{1}(s), g_{2}(s) \in \mathbb{C}(s)$. Selecting $g_{0}(s)=1, g_{1}(s)=0$ and then $g_{0}(s)=0, g_{1}(s)=1$, we obtain two equations:

$$
\left(\begin{array}{cc}
\theta_{1}\left(2 s+\theta_{2}\right) & \left(\theta_{1}-s^{2}\right) \\
2 \theta_{1} & -2 s
\end{array}\right)\left(\begin{array}{l}
\theta_{3} \\
\theta_{4}
\end{array}\right)=-\left(\begin{array}{l}
\mathcal{B}_{3} \\
\mathcal{B}_{4}
\end{array}\right),
$$

with

$$
\begin{aligned}
\mathcal{B}_{3}= & T(s)^{2}\left(Z(s)+s \frac{d Z(s)}{d s}\right) \\
\mathcal{B}_{4}= & s T(s)^{2} \frac{d^{2} Z(s)}{d s^{2}}+T(s)\left(6 s^{2}+4 s \theta_{2}+2 \theta_{1}\right) \frac{d Z(s)}{d s} \\
& +T(s)\left(4 s+2 \theta_{2}\right) Z(s)
\end{aligned}
$$

The above matrix can be easily written in a diagonal form and the expressions for $\theta_{3}$ and $\theta_{4}$ are thus obtained. Notice that they depend on $\theta_{1}$ and $\theta_{2}$.

\section{SIMULATIONS}

Figure 1 shows the simulation results for the estimation of the parameters $\theta_{1}$ and $\theta_{2}$ vs the estimation time. The modified Prony's method (PM) is used as a reference. Each point is obtained by averaging the results over 200 trials. The first plot displays the real part of a sample realization of the noisy signal (1), with $N=1024$ samples and SNR $=20 \mathrm{~dB}$ ). The corresponding value of the constant bias is $\beta=7.9$. The results in the second and third plots show that the estimation is unbiased and insensitive to the constant bias $\beta$ for the presented method (solid line curve). For the same experiment, the results obtained with the modified Prony's method display a bias in the estimates (dot-dashed line curve). Note that even by 

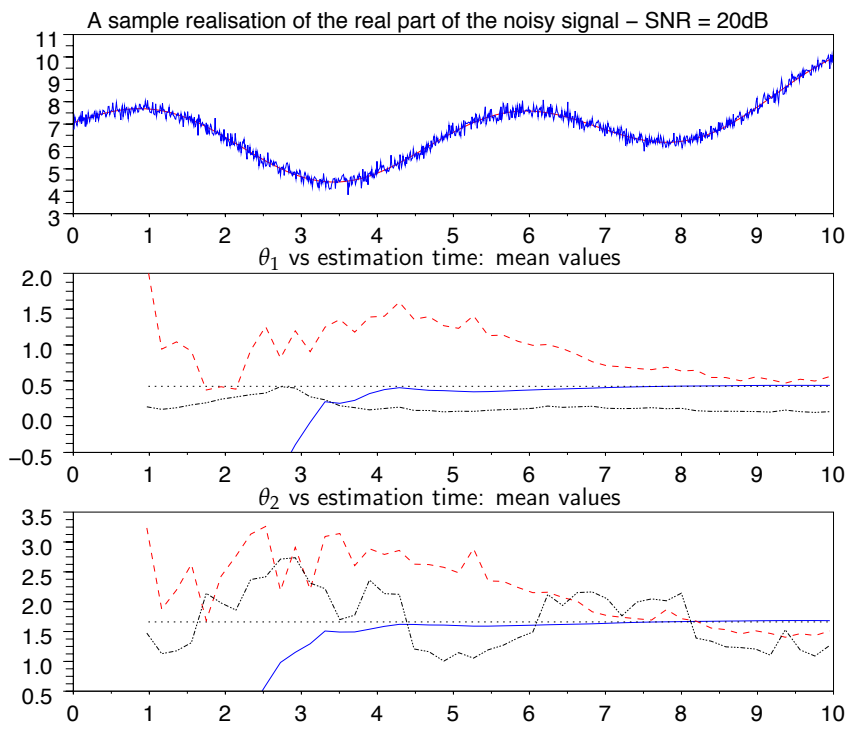

Fig. 1. Comparison with the modified Prony's method: sample mean

setting $\beta=0$ with the Modified Prony's method (dashed line curve), the results of the proposed method still show more robustness to the noise corruption. This is confirmed by the corresponding variances which are displayed below
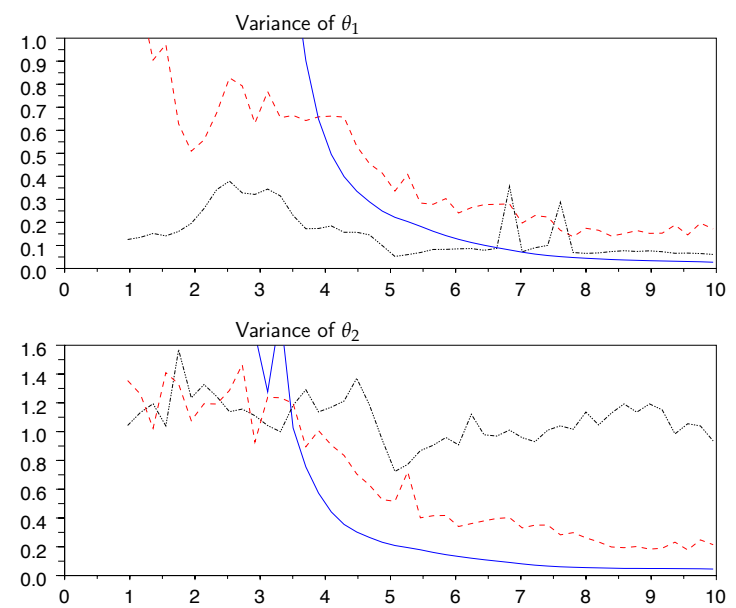

Fig. 2. Comparison with the modified Prony's method: sample variance

\section{CONCLUSION}

The algebraic method we used in this paper proved to be very efficient in this parameter estimation problem of a sum of two sinusoidal waveform signal. Indeed, the two triplets (amplitude, frequency, phase) could be easily identified using the minimal annihilators proposed here.

In the case of a single sinusoidal wave, the results are also convincing (see Ushirobira et al. [2011]).

The perspective of a extension of this algebraic method to a sum of several sinusoidal waveform signal is challenging, but hopefully very likely to be succeeded. A positive result in the three-sinusoid case has already been obtained.

\section{REFERENCES}

S. Bittanti, M. Campi and S. Savaresi, Unbiased estimation of a sinusoid in colored noise via adapted notch filters, Automatica, vol. 33, pp. 209-215, 1997.

S. Bittanti and S. Savaresi, On the parameterization and design of an extended Kalman filter frequency tracker, IEEE Trans. Automat. Control, vol. 45, pp. 1718-1724, 2000.

P. Cartier and Y. Perrin, Integration over finite sets, Nonstandard Analysis in Practice, F. \& M. Diener (Eds), Springer, Berlin, pp. 195-204, 1995.

M. H. Cheng and J. L. Tsai, A new IIR adaptive notch filter, Signal Process, vol. 86, pp. 1648-1655, 2006.

J. Dixmier, Algèbres enveloppantes, Gauthier-Villars, 1974.

M. Fliess, Analyse non standard du bruit, C.R. Acad. Sci. Paris, ser. I, 342, pp. 797-802, 2006.

M. Fliess, Critique du rapport signal à bruit en communications numériques, ARIMA, vol. 9, pp. 419-429, 2008. http://hal .archives-ouvertes.fr/inria-00311719/en/

M. Fliess, C. Join and M. Mboup, Algebraic changepoint detection, Applicable Algebra Engin. Communic. Comput., vol. 21, pp. 131-143, 2010.

M. Fliess, M. Mboup, H. Mounier and H. Sira-Ramírez, Questioning some paradigms of signal processing via concrete examples, in H. Sira-Ramírez, G. Silva-Navarro (Eds.): Algebraic Methods in Flatness, Signal Processing and State Estimation, Editorial Lagares, México, pp. 121, 2003.

http://hal .archives-ouvertes.fr/inria-00001059/en/

M. Fliess and H. Sira-Ramírez, An algebraic framework for linear identification. ESAIM Control Optim. Calc. Variat., vol. 9, pp. 151-168, 2003.

M. Fliess, C. Join, M. Mboup and H. Sira-Ramírez, Compression différentielle de transitoires bruités. C.R. Acad. Sci. Paris, ser. I(339), pp. 821-826, 2004.

M. Fliess and H. Sira-Ramírez, Closed-loop parametric identification for continuous-time linear systems via new algebraic techniques, in H. Garnier \& L. Wang (Eds): Identification of Continuous-time Models from Sampled Data, Springer, pp. 362-391, 2008.

M. Fliess, C. Join and H. Sira-Ramírez, Non-linear estimation is easy. Int. J. Modeling Identif. Control, vol. 4, pp. 12-27, 2008.

http://hal .archives-ouvertes.fr/inria-00158855/en/

S. Haykin, Adaptive Filter Theory (2 ${ }^{\text {nd }}$ ed.), Englewood Cliffs, 1991.

L. Hsu, R. Ortega and G. Damm, A globally convergent frequency estimator, IEEE Trans. Automat. Control, vol. 44, pp. 698-713, 1999.

M. Kahn, M. Mackisack, M. Osborne and G. K. Smyth, On the consistency of Prony's method and related algorithms, J. Comput. Graph. Statist., vol. 1, pp. 329349, 1992.

T. H. Li and B. Kedem, Strong consistency of the contraction mapping method for frequency estimation, IEEE Trans. Inform. Theory, vol. 39, pp. 989-998, 1993.

D. Y. Liu, O. Gibaru and W. Perruquetti, Error analysis for a class of numerical differentiator: application to state observation, 48th IEEE Conference on Decision and Control , China, 2009.

D. Y. Liu, O. Gibaru and W. Perruquetti, Differentiation by integration with Jacobi polynomials, Jour- 
nal of Computational and Applied Mathematics, 2011, doi:10.1016/j.cam.2010.12.023

D. Y. Liu, O. Gibaru and W. Perruquetti, Error analysis of a class of derivative estimators for noisy signals, Numerical Algo., 2011.

M. Mboup, Parameter estimation for signals described by differential equations, Applicable Analysis, vol. 88, pp. 29-52, 2009.

M. Mboup, C. Join and M. Fliess, Numerical differentiation with annihilators in noisy environment, Numerical Algo., vol. 50, pp. 439-467, 2009.

J. C. McConnell and J. C. Robson, Noncommutative Noetherian Rings, Amer. Math. Soc., 2000.

M. Mojiri and A. R. Bakhsahi, An adaptive notch filter for frequency estimation of a periodic signal, IEEE Trans. Automat. Control, vol. 49, pp. 314-318, 2004.

A. Neves, M. Mboup and M. Fliess, An algebraic receiver for full response CPM demodulation, VI Int. Telecom. Symp. (ITS2006), Fortaleza, CE, Brazil, 2006.

http://hal.archives-ouvertes.fr/inria-00086115/en/

A. Neves, M. Miranda and M. Mboup, Algebraic parameter estimation of damped exponentials, EUSIPCO'O'\%, Poznan, Poland, 2007.

http://hal.inria.fr/inria-00179732/en/

M. R. Osborne and G.K. Smyth, A modified Prony algorithm for exponential function fitting, SIAM Journal on Scientific Computing, vol. 16 (1), pp. 119-138, 1995.

P. A. Regalia, Adaptive IIR Filtering in Signal Processing and Control, Marcel Dekker, New York, 1995.

G. M. Riche de Prony, Essai expérimental et analytique : sur les lois de la dilatabilité de fluides élastiques et sur celles de la force expansive de la vapeur de l'eau et de la vapeur de l'alcool à différentes températures, Journal de l'école polytechnique, vol. 1, no. 22, pp. 2476, 1795.

R. Roy and T. Kailath, ESPRIT-estimation of signal parameters via rotational invariance techniques, IEEE Trans. Signal Process, vol. 37, pp. 984-995, 1989.

J. R. Trapero, H. Sira-Ramírez and V. F. Battle, An algebraic frequency estimator for a biased and noisy sinusoidal signal, Signal Processing, vol. 87, pp. 11881201, 2007.

J. R. Trapero, H. Sira-Ramírez and V. F. Battle, A fast on-line frequency estimator of lightly damped vibrations in flexible structures, J. Sound Vibration, vol. 307, pp. 365-378, 2007.

J. R. Trapero, M. Mboup, E. Pereira-Gonzalez and V. B. Feliu, On-line frequency and damping estimation in a single-link flexible manipulator based on algebraic identification, MED, 2008.

J. R. Trapero, H. Sira-Ramírez and V. F. Batlle, On the algebraic identification of the frequencies, amplitudes and phases of two sinusoidal signals from their noisy sum, Int. J. Control, vol. 81, pp. 507-518, 2008.

R. Ushirobira, W. Perruquetti, M. Mboup M. and M. Fliess M., Estimation algébrique des paramètres intrinsèques d'un signal sinusoïdal biaisé en environnement bruité, in GRETSI, Bordeaux, 2011. http://hal archives-ouvertes.fr/inria-00601655/en/

Y. Xiao and Y. Tadokoro, LMS-based notch filter for the estimation of sinusoidal signals in noise, Signal Processing, vol. 46 (2), pp. 223-231, 1995.

\section{Appendix A. FINDING THE ORIGINAL PARAMETERS}

Lemma 15. The parameters $\alpha_{1}, \omega_{1}, \phi_{1}, \alpha_{2}, \omega_{2}, \phi_{2}$ can be obtained from $\Theta_{\text {est }}$.

Proof. From $\theta_{1}$ and $\theta_{2}$ one can easily deduce $\omega_{1}$ and $\omega_{2}$ since $i \omega_{1}$ and $i \omega_{2}$ are the roots of the polynomial $\omega^{2}+$ $\theta_{2} \omega+\theta_{1}=0$. From $\theta_{3}$ and $\theta_{4}$ one obtains $x(0)=-\theta_{3}=$ $\alpha_{1} \exp i \phi_{1}+\alpha_{2} \exp i \phi_{2}$ and $\dot{x}(0)=-\theta_{4}=i\left(\omega_{1} \alpha_{1} \exp i \phi_{1}+\right.$ $\left.\omega_{2} \alpha_{2} \exp i \phi_{2}\right)$ and then easily deduce the remaining parameters $\left(\alpha_{1}, \phi_{1}, \alpha_{2}, \phi_{2}\right)$ :

$$
\begin{aligned}
\left(\begin{array}{c}
-\theta_{3} \\
-\theta_{4}
\end{array}\right) & =\left(\begin{array}{cc}
1 & 1 \\
i \omega_{1} & i \omega_{2}
\end{array}\right)\left(\begin{array}{c}
\alpha_{1} \exp i \phi_{1} \\
\alpha_{2} \exp i \phi_{2}
\end{array}\right) \\
\left(\begin{array}{c}
\alpha_{1} \exp i \phi_{1} \\
\alpha_{2} \exp i \phi_{2}
\end{array}\right) & =\frac{1}{\omega_{2}-\omega_{1}}\left(\begin{array}{c}
-i \theta_{4}-\omega_{2} \theta_{3} \\
i \theta_{4}+\omega_{1} \theta_{3}
\end{array}\right)
\end{aligned}
$$

Taking absolute values and arguments and ordering the frequencies $\omega_{2}>\omega_{1}$ :

$$
\begin{aligned}
\alpha_{1} & =\frac{\sqrt{\left(\Im\left(\theta_{4}\right)-\omega_{2} \Re\left(\theta_{3}\right)\right)^{2}+\left(\Re\left(\theta_{4}\right)+\omega_{2} \Im\left(\theta_{3}\right)\right)^{2}}}{\omega_{2}-\omega_{1}} \\
\tan \left(\phi_{1}\right) & =-\frac{\Re\left(\theta_{4}\right)+\omega_{2} \Im\left(\theta_{3}\right)}{\Im\left(\theta_{4}\right)-\omega_{2} \Re\left(\theta_{3}\right)} \\
\alpha_{2} & =\frac{\sqrt{\left(-\Im\left(\theta_{4}\right)+\omega_{1} \Re\left(\theta_{3}\right)\right)^{2}+\left(\Re\left(\theta_{4}\right)+\omega_{1} \Im\left(\theta_{3}\right)\right)^{2}}}{\omega_{2}-\omega_{1}} \\
\tan \left(\phi_{2}\right) & =-\frac{\Re\left(\theta_{4}\right)+\omega_{1} \Im\left(\theta_{3}\right)}{-\Im\left(\theta_{4}\right)+\omega_{1} \Re\left(\theta_{3}\right)}
\end{aligned}
$$

\section{Appendix B. PROOF OF THEOREM 13}

A $\bar{Q}$-annihilator is of the form (11), that is

$$
\Pi=\sum_{i=0}^{\ell} g_{i}(s) \frac{d^{i+3}}{d s^{i+3}},
$$

where $g_{i}(s) \in \mathbb{C}_{\Theta_{\text {est }}}(s), \forall 1 \leq i \leq \ell$. We apply such annihilator on relation $\mathcal{R}(7)$. Since $\Pi(Q(s))=\Pi(\bar{Q})=$ 0 , we compute $\Pi(P(s) Z(s))$. Using proposition 6 , one obtains:

$$
\frac{d^{i+3}}{d s^{i+3}}(P(s) Z(s))=\sum_{k=0}^{4}\left(\begin{array}{c}
i+3 \\
k
\end{array}\right) P^{(k)}(s) Z^{(i+3-k)}(s)
$$

since $P \in \mathbb{C}[s]$ is of degree 3 . Moreover: $P^{(0)}(s)=s^{3}+$ $\theta_{2} s^{2}+\theta_{1} s, P^{(1)}(s)=3 s^{2}+2 \theta_{2} s+\theta_{1}, P^{(2)}(s)=6 s+2 \theta_{2}$, and $P^{(2)}(s)=6$. Thus using notations (9), $\Pi$ given by (B.1) applied on relation $\mathcal{R}(7)$ reads in the Weyl algebra framework as:

$$
\begin{aligned}
& \sum_{i=0}^{\ell} g_{i}(q)\left(A_{i}(p, q) \theta_{1}+B_{i}(p, q) \theta_{2}+C_{i}(p, q)\right)=0 \\
& \text { where } A_{i}(p, q)=\left(q p+a_{i}\right) p^{i+2}, \\
& B_{i}(p, q)=\left(q^{2} p^{2}+2 a_{i} q p+b_{i}\right) p^{i+1}, \\
& C_{i}(p, q)=\left(q^{3} p^{3}+3 a_{i} q^{2} p^{2}+3 b_{i} q p+c_{i}\right) p^{i},
\end{aligned}
$$

with $a_{i}=(i+3), b_{i}=a_{i}(i+2), c_{i}=b_{i}(i+1), \forall 0 \leq i \leq \ell$. Denote by $L_{i}:=\left[A_{i}(p, q) B_{i}(p, q) C_{i}(p, q)\right]$, then for $i>j$ we have by induction on $(j-i)$ and by example 3 :

$$
L_{j}=p^{(j-i)} L_{i}
$$

and that completes the proof of theorem 13 . 